\title{
The Spatially-Resolved Star Formation History of the M31 Disk from Resolved Stellar Populations
}

\author{
Alexia R. Lewis ${ }^{1}$, Julianne J. Dalcanton ${ }^{1}$, Andrew E. Dolphin ${ }^{2}$, \\ Daniel R. Weisz ${ }^{1,3,4}$ and Benjamin F. Williams ${ }^{1}$, PHAT \\ ${ }^{1}$ Department of Astronomy, University of Washington, Box 351580, Seattle, WA 98195 \\ email: arlewis@astro. washington.edu \\ ${ }^{2}$ Raytheon Company, 1151 East Hermans Road, Tucson, AZ 85756 \\ ${ }^{3}$ University of California Observatories/Lick Observatory, University of California, 1156 High \\ St., Santa Cruz, CA 95064 \\ ${ }^{4}$ Hubble Fellow
}

\begin{abstract}
The Panchromatic Hubble Andromeda Treasury (PHAT) is an HST multi-cycle treasury program that has mapped the resolved stellar populations of $\sim 1 / 3$ of the disk of M31 from the UV through the near-IR. This data provides color and luminosity information for more than 150 million stars. Using stellar evolution models, we model the optical color-magnitude diagram to derive spatially-resolved recent star formation histories (SFHs) over large areas of M31 with $100 \mathrm{pc}$ resolution. These include individual star-forming regions as well as quiescent portions of the disk. With these gridded SFHs, we create movies of star formation activity to study the evolution of individual star-forming events across the disk. We analyze the structure of star formation and examine the relation between star formation and gas throughout the disk and particularly in the 10-kpc star-forming ring. We find that the ring has been continuously forming stars for at least 500 Myr. As the only large disk galaxy that is close enough to obtain the photometry for this type of spatially-resolved SFH mapping, M31 plays an important role in our understanding of the evolution of an $\mathrm{L}^{*}$ galaxy.
\end{abstract}

Keywords. galaxies: evolution - galaxies: individual (M31) - galaxies: spiral - galaxies: structure

\section{Introduction}

The star formation history ( $\mathrm{SFH}$ ) of a galaxy encodes much of the physics of the evolution of that galaxy. It tells us about the evolution of the star formation rate (SFR) throughout the lifetime of the galaxy as well as the evolution of the mass and metallicity distributions.

The SFH can be inferred for bulk populations by fitting the spectral energy distributions (SEDs) of galaxies. The evolution of large samples of galaxies can then be studied by linking local universe galaxies to their likely high redshift progenitors and examining the evolution of the physical properties of the galaxies over time (e.g. Papovich et al. 2011). This technique is advantageous in that it enables a statistically complete analysis of galaxy evolution due to the large number of galaxies in the sample. However, changes to the approximations it requires approximations (SFR as a function of time, dust model, etc.) can have a significant affect on the derived ages and SFRs. It also sacrifices detail for lookback time.

We can perform a more detailed analysis of the evolution of the structure and populations of local universe galaxies by using resolved stellar populations in color-magnitude diagram (CMD) analysis. The CMD contains the history of star formation (SF) and the metallicity distribution of the galaxy. Using individually resolved stars we can probe 
look-back time, allowing us to self-consistently study the evolution of a single galaxy based on its stellar population. With this method, though, we can only study the local universe, which sacrifices lookback time for increased detail.

Using recent observations of the Andromeda Galaxy (M31) as part of the Panchromatic Hubble Andromeda Treasury (PHAT; Dalcanton et al. 2012) we examine the spatiallyresolved SFH of M31 using this 'gold standard' technique. Derivation of the SFH on small scales enables a more detailed comparison of SF with the interstellar medium of a galaxy, helping us uncover clues about the specific conditions that promote SF (e.g. Kennicutt 1998). Historically, this method has been used only to examine the SFHs of low-mass galaxies like the LMC and SMC (Harris \& Zaritsky 2004, 2009). This is the first time it has been used to probe the $\mathrm{SFH}$ of an $\mathrm{L}_{\star}$ galaxy.

\section{Star Formation History Recovery Method}

We use the CMD fitting code MATCH (Dolphin 2002) to derive the SFHs on $~ 100$ pc (projected) scales in the disk of M31. The user specifies ranges to search in age, metallicity, distance, and extinction, as well as an initial mass function and a binary fraction. The code then populates synthetic CMDs, including photometric and completeness errors determined by artificial star tests, and linearly combines them to form many different SFHs. Each of these complex CMDs is compared with the observed CMD and the one that provides the best fit, as determined by a Poisson maximum likelihood technique, is taken to be the model SFH that best describes the data.

To lessen some of the effects of dust on the SFHs, we simplify the fitting process by excluding the redder portions of the CMD from the fit. We model only the main sequence. As a result, we determine only the recent SFH in each region. Our SFHs are reliable back to $\sim 600 \mathrm{Myr}$ ago. This process is repeated in each of our $100 \mathrm{pc}$ regions independently of all other regions. We combine the final results to create maps of SFH in M31.

\section{Results}

We combine the single best fit SFHs in each region into maps of SFR as a function of time. As an ensemble, these reveal the recent SFH of M31 in the PHAT footprint over the last $630 \mathrm{Myr}$. This SFH is shown in Figure 1. The SFHs reveal large-scale, long-lasting, coherent structure in the M31 disk. There are three star-forming rings; a modest inner ring at $\sim 5 \mathrm{kpc}$, the well-known 10 -kpc ring, and an outer, low-intensity ring at $\sim 15 \mathrm{kpc}$ that partially merges with the 10-kpc ring due to a combination of projection effects and a possible warp as visible in HI (e.g. Brinks \& Shane 1984). The 10-kpc ring is visible and actively forming stars throughout the past $600 \mathrm{Myr}$. The ring features at $5 \mathrm{kpc}$ peaked in $\mathrm{SF} \sim 100$ Myr ago but has largely dispersed today. SF in the 15 -kpc feature has increased to the present day, aided in large part by OB 102 which is clearly visible in the maps.

We generate the overall recent SFH within the PHAT footprint by integrating the SFHs of all regions. On the left side of Figure 2 we show the total SFR per time bin over the survey area. The SFR has gradually declined over the last $600 \mathrm{Myr}$, but we do see a peak at $\sim 50$ Myr. Further examination reveals that this peak is not a galaxy-wide feature; instead it is likely due to stellar clusters and OB associations in the rings. On the right side of Figure 2, we plot the average SFR per time bin in regions that fall within the $10-\mathrm{kpc}$ ring and in all other regions. It is very clear the SF in the ring drives the overall $\mathrm{SFH}$ of the disk. The regions outside of the ring show a very low and mostly constant SFR over the last 500 Myr. A more detailed analysis and discussion of the results can be found in Lewis et al. (submitted). 


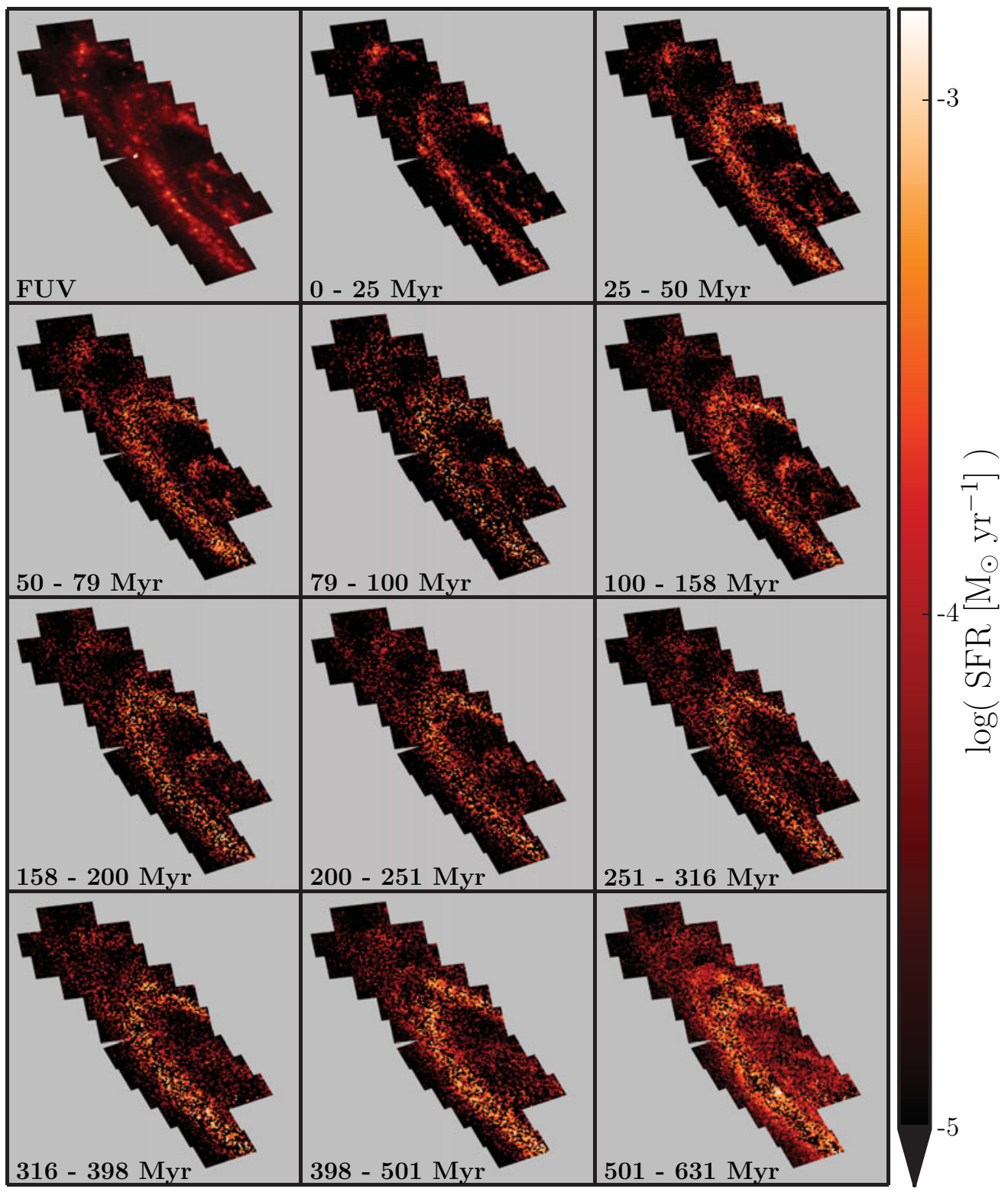

Figure 1. Map of the SFH of M31 covered by the PHAT survey. The pixel value is proportional to the SFR in that region. We have applied a lower cut at a SFR of $10^{-5} \mathrm{M}_{\odot} \mathrm{yr}^{-1}$ in order to highlight structure in the image. The time range is shown in the lower left of each plot. The plot in the top left shows the GALEX FUV image, convolved to the same physical scale as our SFHs for better comparison. The maps are oriented such that north is up and east is left.

\section{Conclusions}

We have measured the spatially-resolved SFH of $\sim 1 / 3$ of the star-forming disk of M31 from HST images as part of the PHAT survey. SF in M31 is largely confined to three ring features, including the well-known 10-kpc ring. These features show elevated levels of SF over the entire reliable lifetime of the SFHs, approximately $600 \mathrm{Myr}$. The 10-kpc ring is long-lived and stationary, producing stars over at least the past $600 \mathrm{Myr}$. It has also 

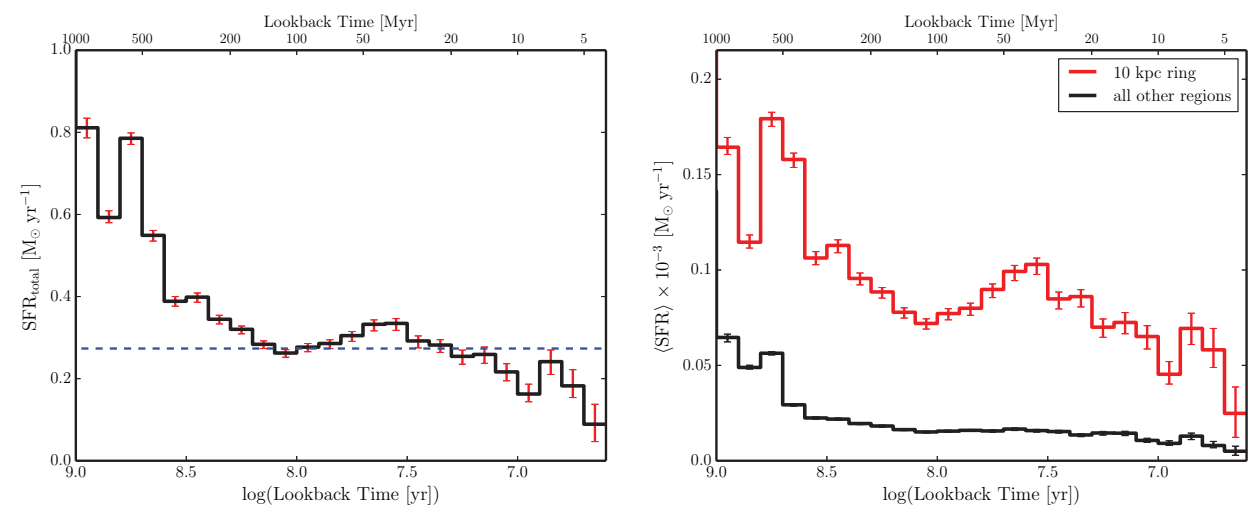

Figure 2. Left:The global SFH of M31 over the last $630 \mathrm{Myr}$, combining the individual SFHs of each field. The dashed line (blue) shows the average SFR over the most recent $100 \mathrm{Myr}(\sim 0.3$ $\mathrm{M}_{\odot} \mathrm{yr}^{-1}$ ). Right: The average SFR per time bin in regions in the 10-kpc ring (top red line) and in all other regions (bottom black line). SF in the ring drives to the recent SFH of M31. In both plots, the error bars are a combination of the random uncertainties and the uncertainties in $\mathrm{A}_{V}$ and $\mathrm{dA}_{V}$. The time bins are $\Delta(\log t)=0.1$. The region in gray indicates the timescale over which the SFHs are not reliable in all regions.

shown little or no significant propagation over the lifetime of these SFHs. Over this time, $\mathrm{SF}$ in the ring has been elevated relative to the surrounding regions and as a result, the ring drives the overall SFH. We have also found that the SFH over the last $500 \mathrm{Myr}$ has been relatively constant, showing a shallow but steady decrease in SFR to the present day with a small burst $\sim 50$ Myr ago.

\section{Acknowledgements}

This project made extensive use of the Stampede supercomputing system at TACC/UT Austin, funded by NSF award OCI-1134872.

\section{References}

Brinks, E. \& Shane, W. W. 1984, A\&AS, 55, 179

Dalcanton, J. J., et al. 2012, ApJS, 200, 18

Dolphin, A. E. 2002, MNRAS, 332, 91

Harris, J. \& Zaritsky, D. 2004, AJ, 127, 1531

Harris, J. \& Zaritsky, D. 2009, AJ, 138, 1243

Kennicutt, R. C., Jr. 1998, ApJ, 498, 541

Papovich, C., Finkelstein, S. L., Ferguson, H. C., Lotz, J. M., \& Giavalisco, M. 2011, MNRAS, 412,1123 